\title{
Soundwalking and Algorithmic Listening
}

\author{
Miguel Carvalhais \\ INESC TEC \& Faculty of Fine Arts, University of Porto \\ Avenida Rodrigues de Freitas, 265 \\ 4049-021 Porto \\ Portugal \\ mcarvalhais@fba.up.pt
}

\author{
Rosemary Lee \\ IT University of Copenhagen \\ Rued Langgaards Vej 7 \\ DK-2300 Copenhagen S \\ Denmark \\ rosl@itu.dk
}

\begin{abstract}
Soundwalking is a listening and composition method that focuses on the exploration of the environment. With roots in the 1970 s, the artistic practices that sprung from soundwalking engage both with the unmediated soundscape as well as with multiple approaches to its augmentation or the augmentation of the human sensory apparatus. Soundwalking emphasises the listener's active and participatory role in the construction of dynamic compositions, shaped as much by the environment as by their presence and actions. Given the increasing relevance of computation in physical and public environments, the omnipresence of the metainterface, and how hybrid environments emerge from physical and virtual spaces, this paper discusses how principles and methodologies of soundwalking may allow the exploration and, ultimately, the understanding of computational environments that are increasingly sonic. This paper explores how in these contexts soundwalking can be used as a poetic and aesthetic resource, leading to the development of a listening that emphasises computation and procedurality, an algorithmic listening.
\end{abstract}

Computational Art, Artificial Aesthetics, Algorithmic Listening, Computational Environments, Procedural Reading.

\section{INTRODUCTION}

Our experience of space has been mediated and transformed by computation: whether in virtual spaces or in physical spaces nonetheless infiltrated by computation. These environments have become hybrids of actual objects in the world and virtual computational objects, becoming an amalgam of the natural acoustic environment - Bernie Krause's geophony and biophony (2008) - of classical anthropophonic sounds, ${ }^{1}$ and of sounds that are produced and experienced through computational systems. This last set of sounds has very particular affordances that significantly impact the soundscape, particularly when we conceptualise it aesthetically: soundscape as how environments are understood by those that inhabit them (Truax 1984, 48).

Environments are hybridised with computation in four principal ways. On one end of the scale, we have fully computational environments, which we may call algorithmic e nvironments. This is the realm of idealised immersive virtual reality, of $c y$ -

\footnotetext{
${ }^{1}$ We may define as classical anthropophonic sounds those that Krause describes in his definition of anthropophony, "all of the human-generated sounds (...) in a given environment: physiological (talking, grunting, body sounds), electromechanical, controlled sound (music, theatre, etc.), and incidental (walking, clothes rustling, etc.)." $(2008,73)$
}

berspace $^{2}$ or the metaverse. ${ }^{3}$ This is the domain of the synthetic and the simulated, where experiences are as removed as possible from the actual, of which not much may remain, with perhaps the exception of human somatic presence and proprioception.

In code/spaces (Kitchin 2011) we find parity with the virtual in a particular "interweaving of computation with the built environment and daily experience" where computation becomes so crucial that "the environment and the experience of it actually ceases to function in the absence of code." (Bridle 2018,37 ) The best current examples of these environments are found in airports or in e-commerce warehouses, but with the growth of home and urban automation, of networked devices, autonomous devices and ubiquitous computing, we expect the number of code/spaces to increase significantly in the future.

Lowering the presence of computation, we arrive to augmented environments, an overlaying of computation to the actual world, where computation and computational devices coexist with the physical in a variety of articulations and hierarchies. This is the domain of augmented reality, but also the space for

\footnotetext{
${ }^{2}$ Popularised by William Gibson's novel Neuromancer (1984)

3 Introduced in Neal Stephenson's novel Snow $C$ rash (1992).
} 
many of our everyday experiences with devices as common as our smartphones.

Lastly, we find that in our post-internet and the post-digital world, there are almost no environments untouched by the effects of computation (Cramer 2013). What we conceptualise as offline environments are also subject to the effects of the computational regime (Hayles 2005, 17) in the "allout internet condition" (Steyerl 2017) that has turned our culture into yet another code/space (Bridle 2018, 38).

\section{COMPUTATIONAL ENVIRONMENTS}

What all these environments have in common is computation. They are defined by processes that obey finitely describable rules (Rucker 2005, 11) and that count and calculate (Berry 2011, 10). Within them, computation is not only used instrumentally, but becomes essential, whether or not it is experienced as such by humans and other agents that inhabit them (Carvalhais 2018a). Inevitably, as it happens in media that have been absorbed by (or displaced to) computational systems, these environments are significantly transformed by computation.

Computation allows the digitalisation of (almost) everything. This in turn gives it the ability to preserve, communicate, and rebuild or simulate information in systems besides those where it was created, and this makes computational systems into excellent remediators. They become a plastic, versatile and protean digital medium (Murray 2012) that now encompasses most of our living and working environments. So, as much as computational systems can continuously breed new med ia (Manovich $2001 ; 2013)$, they are also capable of generating new env ironments. This doesn't happen because their technologies are new but because computation allows environments to be articulated in new ways, and to develop new and unprecedented relations with their inhabitants. Computational media become metamedia, ${ }^{4}$ and computational environments become metaenvironments that are able to develop across multiple and arbitrary forms and modalities. Expanded by computation, environments become more than a space-time where agents exist and become themselves agents. They are now machines in operation, machines that act, process and generate information, and from which computation emerges. Machines

\footnotetext{
${ }^{4}$ Alan Kay called computers the first metamedium, one whose content is "a wide range of already-existing and not-yet-invented media" (Manovich 2013), including even those that "cannot exist physically." (Laurel 1993, 32) Manovich defined metamedia as "the remixing of working methods and techniques of different media within a single project." $(2008,123)$
}

that remediate "the very conditions of being itself" (Galloway 2010).

Computational environments function like languages, and as such, are ergodic. As Christian Andersen and Søren Pold suggested, they are interfaces $(2018,90)$. More than places, they are platforms. As they become omnipresent, they "transcend perception" $(2018,30)$ and, as it becomes increasingly difficult to avoid interacting with them, the more difficult it conversely turns out to understand our role within them and the particular couplings that allow for the production of new signals, information, and knowledge $(2018,35)$.

\section{INTERFACING WITH THE SONIC ENVIRONMENT}

As computational spaces become pervasive and immersive, they also become progressively more acoustic and concrete. They fulfil McLuhan's foretelling of a transition from visual to acoustic spaces (1988, 22) and become holistic and qualitative, breeding complex topologies that embed and envelop all outputs that are produced, whether visual or in any other modality.

In these topologies of "immaterial materiality" (Kwastek 2013, 146) sound is often relegated to a role that is subordinate to visual media. But sound is resilient and pervasive and it seeps in as traces from interaction - such as mechanical keyboard sounds, taps on screens, etc. - as designed artificial interaction sounds - such as the keyboard clicks on mobile devices, or other elements of sonic interaction design ${ }^{5}$ (Erkut 2016) - and as sounds produced by the hardware itself - such as refrigeration fans in computers, electric hums, etc.

And computation listens to us. For a long time, sound was almost exclusively limited to be an output of computational systems. Sound gave computational devices voices and the ability to communicate with the world around them, to announce their presence within a larger context (Breinbjerg 2008, 248-9), but it was incidental. Nowadays sound is becoming commonly used as an input, as a medium for interaction with systems that need to listen to their environments. When we use voice commands to interact with virtual assistants, when we dictate text to a device, or Shazam a piece of music, we ask our computers to actively listen to us and to our environments.

To be able to operate and navigate in these environments, we need to be capable to read them and to deduce procedurality and computation (Carvalhais, 2017; Carvalhais and Cardoso 2018a; $2018 b)$. As sound is a part of the computational, it becomes a fundamental part of the process of

\footnotetext{
${ }^{5}$ Such as contextual sounds of alerts and notifications.
} 
reading computational environments, a process that needs to go beyond the visual and spatial to embrace the acoustic and the sequential (Coupland $2011,189)$. More than hearing, one needs to develop listening as a skill (Bosma 2011, 192) and to reflectively listen to computational environments. We need to engage with computation in acts of active and participatory listening.

\section{LISTENING AND MOVING}

We inhabit these environments. We listen to them. We move in them. Listening is never static and is closely related to movement in the mechanics of sound perception and production.

Not only does our perception move its attention through the multitude of sounds that it encounters in the environment, but it also shifts from those to our inner sound world and thoughts and back again to the outside. (Westerkamp 2017, 30)

What does movement mean once we find ourselves in an environment that transcends physical space? If the presence in actual environments may be passive, not entailing acting on them in any way, when we find ourselves in any computational environment, we discover a context of constant acting (Cardoso 2014).

As Janet Murray noted (1997; 2012), we conceptualise computational environments in spatial terms, attributing them particular geographies and toponymy. ${ }^{6}$ But this is far more than just a conceptual issue, because when interacting with a computational environment, what happens to us is causally linked to computational events. The outputs of these are not simply recorded signs of past events - as would be the case with classical media - but actual events happening in the computational system "now, and, unlike the action on the stage of a theater, (...) happening to you." $(1997,81)$ Realtime events happen in a particular space-time and, therefore, computational processes are not analogous and interchangeable, but are always very concrete occurrences that happen here and now to a particular person that interacts with the system in a particular context.

In interactive systems we may posit that humans become an integral part of the computation, ${ }^{7}$ and that their somatic topologies therefore become a part of the topology of the system. This makes computational systems become partially embodied,

\footnotetext{
${ }_{7}^{6}$ By e.g. using such names as "cyberspace".

${ }^{7}$ We may define as interactive those systems that accept any external inputs, either by human users or by any other object. A human interactor becomes an agent in the programming of the computational system because every interaction with the system can be seen as an act that influences and determines its program.
}

with their space enmeshing with that of the human in an ergodic superposition of virtual and actual where algorithms, code, and computational machines are as relevant as human interaction and speculative imagination (Cramer 2005). And in both virtual and actual environments, the participative role of the listener while actively navigating and modulating their own experience of the soundscape is key.

We have been studying the inference of computational processes from the reading of artworks ${ }^{8}$ in an effort to understand the uniqueness of the aesthetics of computation and of its poietic affordances. Computational environments are time-based, they are contexts for the composition of events in time, regardless of whether or not sound is used (Zielinski 2006, 271). Therefore, perhaps listening should not specifically emphasise the modality of sound but rather the perception of time-based processes and transient phenomena that are key to reading computation. As such, listening can be used to understand the processes of the computationalisation of environments and to develop interobjective relations within them (Morton 2013, 81).

\begin{abstract}
Sight isolates, sound incorporates. Whereas sight situates the observer outside what he views, at a distance, sound pours into the hearer. Vision dissects, as Merleau-Ponty has observed (1961). Vision comes to a human being from one direction at a time: to look at a room or a landscape, I must move my eyes around from one part to another. When I hear, however, I gather sound simultaneously from every direction at once: I am at the center of my auditory world, which envelopes me, establishing me at a kind of core of sensation and existence. (...) You can immerse yourself in hearing, in sound. There is no way to immerse yourself similarly in sight. (Ong 1982, 71)
\end{abstract}

\section{SOUNDWALKING}

Soundwalking is a listening and composition method that focuses on the exploration of the environment. It is a well-established practice with a large repertoire of works and a terminology that grew from its pioneers to contemporary sound artists. Artistic practices that sprung of soundwalking engage both with the unmediated soundscape as with multiple approaches to the augmentation of the human sensorium, using technology to amplify sound or to sonify information and allow the perception of phenomena that may otherwise be impossible to sense directly.

Soundwalking essentially entails moving in space while focusing one's attention on the sonic environment. R. Murray Schafer describes it as an ex-

\footnotetext{
${ }^{8}$ See Carvalhais (2016; 2019), Carvalhais and Cardoso (2018a), Lee (2019), and Lee and Carvalhais (2019).
} 
ploration of a soundscape guided by a score, that is often a map, that draws one's attention to particular sounds and ambiences to be found along the way (1977, 213).

Soundwalking enhances the listener's capacity to engage with works while simultaneously opening up a much broader range of interpretations and roles, because their role may be equated to that of an audience when instructed to listen to the soundscape, or, when asked to participate with the soundscape, their role becomes that of a "composer-performer." (Schafer 1977, 213)

Soundwalking is a form of active and participatory listening where the listener engages with the environment through moving and acting in it. It is a methodology for the active interpretation of the environment. As it places the listener in the environment, it inevitably makes them become part of the soundscape, contributing to the development of a heightened awareness of their soundmaking in the environment and, consequently, of all soundmaking. The act of listening thus becomes ecological.

Soundwalks give us that chance to practice listening to the unravelling of that continuous now, as we are grounded in the movement of walking and at the same time are learning to be mindful about our own soundmaking in this world. (Westerkamp 2017, 37)

The soundscape, as defined by Schafer, is the sonic environment that is explored, or any portion of it one may focus upon. Importantly, this "term may refer to actual environments, or to abstract constructions such as musical compositions and tape montages, particularly when considered as an environment." (1977, 274) Within a soundscape one perceives keynote sounds and sound signals. Keynote sounds are named after the notes that identify the tonality of a composition, and describe the anchor sounds in an environment. They do not have to be listened to consciously, but they are present continuously or with a high-enough frequency "to form a background against which other sounds are perceived. Examples might be the sound of the sea for a maritime community or the sound of the internal combustion engine in the modern city." (1977, 272) Keynote sounds act as framers and as "conditioning agents" for the perception of other sounds, so they are likened "to the ground in the figureground grouping of visual perception." (1977, 272) Without contrasting with the ground developed by keynote sounds, foreground sounds would not be possible.

The foreground sounds that are listened to consciously are the sound signals. Although any sound can be listened to consciously, thus becoming figure instead of ground, Schafer defines sound signals as those that have to be listened to $(1977,10)$, because they constitute acoustic warning devices or because they encode information directed at the listener. Within sound signals, one may further understand soundmarks, a term that Schafer derives from landmark and that refers to a sound that is unique in a given community or environment and that possesses qualities that make it become especially regarded or noticed in that context.

When Pierre Schaeffer defined the acousmatic (2012), he described a mode of listening he termed of reduced or concentrated listening. This removal of the sound sources, the freeing of sounds that acousmatic music proposes, turns listening around. If doesn't necessarily remove causality from sounds but rather reinforces and reframes causality in the way as it forces one to always listen to sounds together and understand them in relation to each other (Larivière 2019, 81). Reduced listening depends on a two-part acousmatic reduction, "the first taking place through the removal of visual cues, and the second through the intentional disregard of the perceived sources and origins of a sound." (Demers 2010, 27) Actual soundscapes are sometimes acousmatic in the first sense, because sound sources may be invisible or inaccessible. Computational soundscapes are often acousmatic in both senses because they also enforce the second reduction, and because they are able to confront us with truly unrecognizable sounds (Demers 2010, 39). The acousmatic condition is then one of interobjectivity and emergence of sound events and sound objects.

Sound events and sound objects are the smallest self-contained particles of a soundscape, as defined by human hearing (Schafer 1977, 274). Sound objects were defined by Schaeffer as abstract acoustical objects, phenomenological sound formations that are independent of their referential qualities. Contrasting with this, Schafer defines sound events as symbolic, semantic or structural objects for study, as "nonabstractable point of reference, related to a whole of greater magnitude than itself." (1977, 274) In the acousmatic condition, sounds are perceived sensually, causally, ecologically, and in context. For the soundscape and the soundwalks within it, context is everything, and understanding as much as possible of it contributes to transforming both our listening and comprehension of what is listened to, while at the same time making it abundantly clear that we are never able to "be aware of everything at all times." (Westerkamp 2017, 30)

As such, the conceptual framework of soundwalking can also be an effective resource to think about our actions within computational environments, and a good methodology to understand the type of augmented and spatial listening that incorporates actual, physical spaces and virtual environments. 


\section{ALGORITHMIC LISTENING}

In light of this, we can ask: 1) Can the principles of soundwalking can be used to explore and interpret computational environments? 2) Can the fundamental ideas in soundwalking gain new relevance in these new computational environments? 3) Can they ultimately contribute to a form of listening that emphasises computation and procedurality, an algorithmic listening?

As in soundwalking, when inhabiting a computational environment, we enter a process of just-intime composition through which we make sense of our experiences. This is a process in which we are conflated with computation and coupled with the environment to produce new aesthetic objects and experiences (Harman 2018, 105). This happens in all environments where computation is present, environments that function as interfaces that have to be explored as topological spaces. While we traverse them, we discover horizons of action, and form horizons of intent (Upton 2015) within their phase-spaces. Although this process is very often visual, sound - either by itself or in the context of audiovisual forms - is an increasingly important component in these processes, as it is for our sense of space and for how we map our environment. In voice-driven and other sonic interactions, it may even act as a main driver of the experience and as a fundamental medium for the development of the computational system's technological umwelt. $^{9}$

The goals of algorithmic listening are not only aesthetic. The listener does not develop just-in-time composition for its own sake but also because this allows them to probe the system and to discover and explore the processes at its core. Algorithmic listening emphasises computation and procedurality and has resemblances with the algorithmic gaze described by Boris Groys, ${ }^{10}$ a gaze that also turns around and looks from within the artwork to its world $(2016,20)$.

Algorithmic listening tries to discover the spatial and temporal boundaries of an environment. Lis-

\footnotetext{
${ }^{9}$ Expanding upon Jakob von Uexküll (1934) on the “Umwelt”, describing an organism's perceptual relation to its environment, Rosemary Lee (2008) uses the term "technological umwelt" to describe technologicallymediated perceptual relations between an actor and its environment.

${ }^{10}$ Stephen Graham (2006) has used the term "algorithmic gaze" in relation to visual warfare technologies, in a sense that is much closer to Harun Farocki's operative images, a term that is also used by Trevor Paglen (2014) and others. Frieder Nake has also suggested the term as a way of expressing not the visual but rather the mental process of programming computers (Andersen and Pold 2018, 110).
}

tening then becomes a hybrid of human and computational operations, it becomes interobjective, with the ear guided by logic and technology to navigate through computational environments where listeners are positioned and set free to dwell in.

\section{ACKNOWLEDGMENTS}

This work is financed by National Funds through the Portuguese funding agency, FCT - Fundação para a Ciência e a Tecnologia within project: UID/EEA/50014/2019.

\section{REFERENCES}

Andersen, C. U. \& Pold, S. B. (2018) The Metainterface: The Art of Platforms, Cities, and Clouds. Cambridge, MA: The MIT Press.

Berry, D. M. (2011) The P hilosophy of S oftware: Code a nd Mediation in $t$ he D igital $A$ ge. Basingstoke: Palgrave Macmillan.

Bosma, J. (2011) Nettitudes: Let 's T alk $N$ et $A$ rt. Rotterdam: NAi Publishers.

Breinbjerg, M. (2008) System Event Sounds. In Software Studies: A Lexicon. Cambridge, MA: The MIT Press.

Bridle, J. (2018) New D ark A ge: T echnology and the End of the Future. London: Verso.

Cardoso, P. \& Carvalhais, M. (2014) Transcoding Action: Embodying the game. In ISEA 2014. Dubai.

Carvalhais, M. (2016) Artificial Aesthetics: Creative Practices in Computational Art and Design. Porto: U.Porto Edições.

Carvalhais, M. (2019) Breaking the Black Box: Procedural Reading, Creation of Meaning and Closure in Computational Artworks. In Artificial Intelligence in A rts, $M$ usic and $D$ esign. Berlin: Springer.

Carvalhais, M. \& Cardoso, P. (2017) Creation of Meaning in Processor-Based Artefacts. In ISEA 2017 Bio-Creation and Peace. Manizales: Department of Visual Design, Universidad de Caldas.

Carvalhais, M. \& Cardoso, P. (2018a) Empathy in the Ergodic Experience of Computational Aesthetics. In ISEA 2018 Intersections. Durban: Faculty of Arts and Design, Durban University of Technology.

Carvalhais, M. \& Cardoso, P. (2018b) Narrative Games in Ergodic Media. Communication Studies, 2, 55-65.

Coupland, D. (2011) Marshall McLuhan: You Know Nothing of My Work! New York, NY: Atlas \& Co. 
Cramer, F. (2005) Words Made F lesh: Code, Culture, I magination. Rotterdam: Piet Zwart Institute.

Cramer, F. (2013) What is 'Post-digital'? Available from http://www.aprja.net/?p=1318 (2 September 2019).

Demers, J. (2010) Listening Through the Noise: The Aesthetics of Experimental Electronic Music. New York, NY: Oxford University Press.

Erkut, C. \& Serafin, S. (2016) From Ecological Sounding Artifacts Towards Sonic Artifact Ecologies. CHI 2016. San Jose, CA.

Galloway, A. R. (2010) The Anti-Language of New Media. Discourse, 32, 276-284.

Graham, S. (2006) Interrupting the Algorithmic Gaze? $U$ rban $W$ arfare and $U$ S Military Technology Available from https://www.academia.edu/2887853/Interrupting _the_algorithmic_gaze_Urban_warfare_and_US _military_technology (2 September 2019).

Groys, B. (2016) In the Flow. London: Verso.

Harman, G. (2018) Object-Oriented Ontology: A New $T$ heory of $E$ verything. London: Pelican Books.

Hayles, N. K. (2005) My Mother Was a Computer: Digital Subjects and Literary Texts. Chicago, IL: The University of Chicago Press.

Kitchin, R. \& Dodge, M. (2011) Code/Space: Software and Everyday Life. Cambridge, MA: The MIT Press.

Krause, B. (2008) Anatomy of the Soundscape: Evolving Perspectives. Journal of $t$ he Audio Engineering Society, 56, 73-80.

Kwastek, K. (2013) Aesthetics of I nteraction in Digital Art. Cambridge, MA: The MIT Press.

Larivière, R. R. (2019) Acousmatic Recreations. In Composer I 'écoute /C omposing L istening. Rennes: Shelter Press.

Laurel, B. (1993) Computers as Theatre. Reading, MA: Addison-Wesley.

Lee, R. (2018) The Limits of Algorithmic Perception: Technological Umwelt. In Politics of the Machines: Art and After. Copenhagen.

Lee, R. (2019) Aesthetics of Uncertainty. In $x$ CoAx 2019: $P$ roceedings of the $S$ eventh Conference on Computation, Communication, Aesthetics, and $X$. Milan.

Lee, R. \& Carvalhais, M. (2019) Net Art and the Performance of Images. Presented in The Web that $W$ as: $A$ rchives, Traces, $R$ eflections. University of Amsterdam.
Manovich, L. (2001) The Language of New Media. Cambridge, MA: The MIT Press.

Manovich, L. (2008) Import / Export. In Software Studies: A Lex icon. Cambridge, MA: The MIT Press.

Manovich, L. (2013) Software Takes Command: Extending the Language of New Media. New York, NY: Bloomsbury Academic.

McLuhan, M. \& McLuhan, E. (1988) Laws of the Media: The New Science. Toronto: University of Toronto Press.

Morton, T. (2013) Hyperobjects: Philosophy and Ecology after the End of the World. Minneapolis, MN: University of Minnesota Press.

Murray, J. H. (1997) Hamlet on the Holodeck: The Future of Narrative in C yberspace. Cambridge, MA: The MIT Press.

Murray, J. H. (2012) Inventing t he Medium: Principles of Interaction Design as a Cultural Practice. Cambridge, MA: The MIT Press.

Ong, W. J. (1982) Orality and L iteracy. London: Routledge.

Paglen, T. (2014) Operational Images. E-flux 59.

Rucker, R. (2005) The Lifebox, the Seashell, and the Soul: What G narly Computation Taught Me About Ultimate Reality, the Meaning of Life, and How to Be Happy. New York, NY: Thunder's Mouth Press.

Schaeffer, P. (2012) In Search of a C oncrete Music. Berkeley, CA: University of California Press.

Schafer, R. M. (1977) The Soundscape: Our Sonic Environment and the Tuning of the World. Rochester, VT: Destiny Books.

Steyerl, H. (2017) Duty Free Art: Art in the Age of Planetary Civil War. London: Verso Books.

Truax, B. (1984) Acoustic C ommunication. Norwood, NJ: Ablex.

Upton, B. (2015) The Aesthetic of Play. Cambridge, MA: The MIT Press.

von Üexküll, Jakob. (1934). A Stroll through the Worlds of Animals and Men: A Picture Book of Invisible Worlds. In Semiotica (1992) 89 (4): 319-91.

Westerkamp, H. (2017) The Practice of Listening in Unsettled Times. In Proceedings of I nvisible Places: Sound U rbanism and Sense of $P$ lace. Ponta Delgada.

Zielinski, S. (2006) Deep $T$ ime of $T$ he Media: Toward a $n$ A rchaeology of Hearing a nd Seeing by Technical Means. Cambridge, MA: The MIT Press. 